\title{
ON THE REPRESENTATION THEOREM BY THE LAPLACE TRANSFORMATION OF VECTOR-VALUED FUNCTIONS
}

\author{
ISAO MiYADERA \\ (Received February 18, 1956)
}

1. Introduction. The theory of the Laplace integral of vector-valued functions has been developed by E. Hille [2]. But, there is no theorem giving conditions that a vector-valued function is represented as the Laplace transformation of a function in $B_{p}([0, \infty) ; X)$.

P. G. Rooney [4] has recently developed this representation theory in terms of an inversion operator given by the formula

$$
L_{k}, t[f]=\left(k e^{2 k} / \pi t\right) \int_{0}^{\infty} s^{-\frac{1}{2}} \cos \left(2 k s^{\frac{1}{2}}\right) f(k(s+1) / t) d s,
$$

where the integral is Bochner integral.

Since Rooney's method is quite general, his argument can be used for Widder's operator given by the formula

$$
L_{k}, t[f]=\frac{(-1)^{k}}{k !} f^{(k)}(k / t)(k / t)^{k+1},
$$

where $f^{(k)}(t)$ denotes the $k$-th strong derivative of $f(t)$.

In his argument, the basic space $X$ is a reflexive Banach space for $1<p<\infty$ and is a uniformly convex Banach space for $p=\infty$.

The main purpose of the present paper is to give a necessary and sufficient condition in order that a function $f(s)$ is the Laplace transformation of a function in $B_{\infty}([0, \infty) ; X)$ where $X$ is a reflexive Banach space. The representation theorems are stated in terms of the Widder operator (1. 2) and we can obtain the theorems similarly as in the numerically-valued case.

2. Preliminary theorems. Let $X$ be a Banach space.

Definition. A vector-valued function $f(s)$ on $(0, \infty)$ into $X$ is said to belong to $B_{p}([0, \infty) ; X)(1 \leqq p<\infty)$ if $f(s)$ is Bochner measurable and

$$
\int_{0}^{\infty}\|f(s)\|^{p} d s<\infty .
$$

Similarly $f(s)$ is said to belong to $B_{\infty}([0, \infty) ; X)$ if it is Bochner measurable in $(0, \infty)$ and $\|f(s)\|$ is bounded except in a null set.

It is obvious that the class $B_{p}([0, \infty) ; X)$ becomes a Banach space under the norm 


$$
\|f(\cdot)\|_{p}=\left\{\int_{0}^{\infty}\|f(s)\|^{p} d s^{\mid 1 / p}(1 \leqq p<\infty),\|f(\cdot)\|_{\infty}=\operatorname{ess} \sup \|f(s)\| .\right.
$$

The following inversion formula has been proved by E. Hille [2, Theorem 10.3.4]:

Theorem 1. If $\varphi(t)$ is in $B_{1}([0, \omega] ; X)$ for each $\omega>0$, and if the integral

$$
f(s)=\int_{0}^{\infty} e^{-s t} \varphi(t) d t
$$

converges for some $s$, then

$$
\lim _{k \rightarrow \infty} L_{k}, t[f]=\varphi(t)
$$

in the Lebesgue set of $\varphi(t)$.

The following theorem which is fundamental in the representation theory, is proved similarly as in the numerically-valued case, so that we omit the details.

THEOREм 2. If for each positive integer $k$

$$
\left\|\int_{0}^{s} L_{k}, t[f] d t\right\|=O(s) \quad(s \rightarrow \infty),
$$

then $f(\infty)$ exists and

$$
\lim _{k \rightarrow \infty} \int_{0}^{\infty} e^{-s t} L_{k}, t[f] d t=f(s)-f(\infty) \quad(0<s<\infty) .
$$

3. Representation of vector-valued functions by Laplace transformations of functions in $B_{p}([0, \infty) ; X), 1<p<\infty$.

THEOREM 3. If $X$ is a reflexive Banach space, then a necessary and sufficient condition that $f(s)$ can be expressed in the form

$$
f(s)=\int_{0}^{\infty} e^{-s t} \varphi(t) d t \quad(s>0),
$$

where $\varphi(t) \in B_{p}([0, \infty) ; X)$, p fixed, $1<p<\infty$, is that

(i) $f(s)$ has strong derivatives of all orders in $0<s<\infty$ and $f(\infty)=0$,

(ii) there exists a consiant $M$ such that

$$
\left(\int_{0}^{\infty}\left\|L_{k}, t[f]\right\|^{p} d t\right)^{1 / p} \leqq M \quad(k=1,2, \ldots) .
$$

Proof of Necessity. Suppose

$$
f(s)=\int_{0}^{\infty} e^{-s t} \varphi(t) d t \quad(s>0),
$$

and $\varphi(t) \in B_{p}([0, \infty) ; X)$. Then using Hölder's inequality we have 


$$
\|f(s)\| \leqq \int_{0}^{\infty} e^{-s t}\|\varphi(t)\| d t \leqq\left(\int_{0}^{\infty}\|\varphi(t)\|^{p} d t\right)^{1 / p}(s q)^{-1 / q},
$$

where $1 / p+1 / q=1$, so that (i) is necessary. Another application of Hölder's inequality gives

$$
\begin{aligned}
& \left\|L_{k, t}[f]\right\|^{p}=\left\|\int_{0}^{\infty} e^{-k u / t} \frac{u^{k}}{k !}\left(\frac{k}{t}\right)^{k+1} \varphi(u) d u\right\|^{p} \\
& \leqq \int_{0}^{\infty} e^{-k u / t} \frac{u^{k}}{k !}\left(\frac{k}{t}\right)^{k+1}\|\varphi(u)\|^{p} d u\left(\int_{0}^{\infty} e^{-k u / t} \frac{u^{k}}{k !}\left(\frac{k}{t}\right)^{k+1} d u\right)^{p / u} \\
& \leqq \int_{0}^{\infty} e^{-k u / t} \frac{u^{k}}{k !}\left(\frac{k}{t}\right)^{k+1}\|\varphi(u)\|^{p} d u
\end{aligned}
$$

so that

$$
\begin{aligned}
\int_{0}^{\infty}\left\|L_{k, t}[f]\right\|^{p} d t & \leqq \int_{0}^{\infty}\|\varphi(u)\|^{p} u^{k} d u\left(\int_{0}^{\infty} e^{-k u / t} \frac{1}{k !}\left(\frac{k}{t}\right)^{k+1} d t\right) \\
& =\int_{0}^{\infty}\|\varphi(u)\|^{p} d u .
\end{aligned}
$$

Hence (ii) is necessary.

Proof of Sufficiency. By (ii) and Hölder's inequality

$$
\int_{0}^{s}\left\|L_{k}, t[f]\right\| d t \leqq M s^{(p-1) / p} \leqq M s
$$

for every $s>1$ and for each positive integer $k$. Thus we obtain by Theorem 2 and (i)

$$
\lim _{k \rightarrow \infty} \int_{0}^{\infty} e^{-s t} L_{k}, t[f] d t=f(s) \quad(0<s<\infty) .
$$

Since $X$ is a reflexive Banach space, $B_{p}([0, \infty) ; X), 1<p<\infty$, is reflexive (see S. Bochner and A.E. Taylor [1] and B. J. Pettis [3]). Therefore $B_{p}([0$, $\infty) ; X$ ) is locally weakly compact. Since

$$
\left(\int_{0}^{\infty}\left\|L_{k},[f]\right\|^{p} d t\right)^{1 / p} \leqq M
$$

there exists an element $\varphi(t)$ of $B_{p}([0, \infty) ; X)$ and an increasing sequence $\left\{k_{i}\right\}$ of positive integers such that for every $y^{*}$ in $B_{p}^{*}([0, \infty) ; X)$

$$
\lim _{k_{i} \rightarrow \infty} y^{*}\left(L_{k_{i}}, \cdot[f]\right)=y^{*}(\varphi(\cdot)) .
$$

Let $x^{*}$ be an arbitrary element of $X^{*}$. Then if $g(t)$ is an arbitrary element of $B_{p}([0, \infty) ; X)$, 


$$
x *\left(\int_{0}^{\infty} e^{-s t} g(t) d t\right)=y_{s}^{*}(g(\cdot))
$$

defines an element in $B_{p}^{*}([0, \infty) ; X)$ for each $s>0$. Thus we have

$$
\begin{aligned}
y_{s}^{*}(\varphi(\cdot)) & =x^{*}\left(\int_{0}^{\infty} e^{-s t} \varphi(t) d t\right)=\lim _{k_{i} \rightarrow \infty} y_{s}^{*}\left(L_{k_{i}}, .[f]\right) \\
& =\lim _{k_{i} \rightarrow \infty} x^{*}\left(\int_{0}^{\infty} e^{-s t} L_{k_{i}}, t[f] d t\right)
\end{aligned}
$$

so that by (3. 1)

$$
x^{*}(f(s))=x^{*}\left(\int_{0}^{\infty} e^{-s t} \varphi(t) d t\right) .
$$

Hence

$$
f(s)=\int_{0}^{\infty} e^{-s t} \varphi(t) d t \quad(0<s<\infty)
$$

and the theorem is proved.

4. Representation of vector-valued functions by Laplace transformations of functions in $B_{\infty}([0 ; \infty) ; X)$.

The following Lemma is due to P. G. Rooney [4]:

Lemмa. If $\left\{T_{\sigma} ; 0<\sigma<\infty\right\}$ is a set of bounded linear operators on a separable Banach space $X$ into a reflexive Banach space $Y$, and if $\left\|T_{\sigma}\right\| \leqq M$ independently of $\sigma$ for all $\sigma>0$, then there exists an increasing unbounded sequence $\left\{\sigma_{i}\right\}$ and a linear operator $T$ on $X$ into $Y$ with $\|T\| \leqq M$, such that

$$
\lim _{\sigma_{i} \rightarrow \infty} y^{*}\left(T_{\sigma_{i}}(x)\right)=y^{*}(T(x))
$$

for every $x$ in $X$ and every $y^{*}$ in $Y^{*}$.

TheOREM 4. If $X$ is a reflexive Banach space, then a necessary and sufficient condition that $f(s)$ can be expressed in the form

$$
f(s)=\int_{0}^{\infty} e^{-s t} \varphi(t) d t \quad(s>0),
$$

where $\varphi(t) \in B_{\infty}([0, \infty) ; X)$, is that

(i') $f(s)$ has strong derivatives of all orders in $0<s<\infty$,

(ii') there exists a constant $M$ such that for $0<s<\infty$

$$
\frac{s^{k+1}}{k !}\left\|f^{(k)}(s)\right\| \leqq M \quad(k=0,1,2, \ldots) .
$$

Proof of Necessity. Suppose 


$$
f(s)=\int_{0}^{\infty} e^{-s t} \varphi(t) d t \quad \quad(s>0)
$$

and $\varphi(t)$ is in $B_{\infty}([0, \infty) ; X)$. Then $\left(i^{\prime}\right)$ is obvious. Since

$$
\frac{s^{k+1}}{k !}\left\|f^{(k)}(s)\right\| \leqq \frac{s^{k+1}}{k !} \int_{0}^{\infty} t^{k}\|\varphi(t)\| e^{-s t} d t \leqq \operatorname{ess} \sup \|\varphi(t)\|,
$$

(ii') is necessary.

Proof of Sufficiency. By (i') and (ii')

and for $0<t<\infty$

$$
t\|f(t)\| \leqq M
$$

so that $f(\infty)=0$ and

$$
\left\|L_{k}, t[f]\right\| \leqq M
$$

$$
(k=1,2, \ldots)
$$

$$
\left\|\int_{0}^{s} L_{k}, t[f] d t\right\|=O(s)
$$

Thus we have by Theorem 2

$$
\lim _{k \rightarrow \infty} \int_{0}^{\infty} e^{-s t} L_{k}, t[f] d t=f(s)
$$$$
(0<s<\infty)
$$

Let $\psi(t)$ be in $L_{1}(0, \infty)$. Define

$$
T_{k}(\psi)=\int_{0}^{\infty} \psi(t) L_{k}, t[f] d t .
$$

It is obvious that $\left\{T_{k}\right\}$ is a set of bounded linear operators on a separable Banach space $L_{1}(0, \infty)$ into a reflexive Banach space $X$ and $\left\|T_{k}\right\| \leqq M$. Thus, by the preceding lemma, there exists an increasing sequence $\left\{k_{i}\right\}$ of positive integers and a bounded linear operator $T$ on $L_{1}(0, \infty)$ into $X$ with $\|T\| \leqq M$, such that for every $x^{*}$ in $X^{*}$ and every $\psi$ in $L_{1}(0, \infty)$,

$$
\lim _{k_{i} \rightarrow \infty} x^{*}\left(T_{k_{i}}(\psi)\right)=x(T(\psi)) \text {. }
$$

Let $\omega$ be an arbitrary positive integer. Since $B_{2}([0, \omega] ; X)$ is reflexive and

$$
\left(\int_{0}^{\omega}\left\|L_{k_{i}}, t[f]\right\|^{2} d t\right)^{1 / 2} \leqq M \omega^{1 / 2}
$$

there exists an element $\varphi^{(\omega)}(t)$ of $B_{2}([0, \omega] ; X)$ and a sequence $\left\{k_{i}{ }^{\prime}\right\}\left(\subset\left\{k_{i}\right\}\right)$ such that for every $y_{(\omega)}^{*}$ in $B^{*}([0, \omega] ; X)$

$$
\lim _{k_{i}^{\prime} \rightarrow \infty} y_{(\omega)}^{*}\left(L_{k_{i}^{\prime}}, .[f]\right)=y_{(\omega)}^{*}\left(\varphi^{(\omega)}(\cdot)\right) .
$$

Let $x^{*}$ be an arbitrary element of $X^{*}$. Then if $\psi(t)$ is an arbitrary 
Lebesgue measurable function such that $\int_{0}^{\omega}|\psi(t)|^{2} d t<\infty$ and $\psi(t)=0$ for $t>\omega$, and if $g(t)$ is an arbitrary element of $B_{\mathfrak{2}}([0, \omega]: X)$, then

$$
x *\left(\int_{0}^{\omega} \psi(t) g(t) d t\right)
$$

defines an element in $B_{2}^{*}([0, \omega] ; X)$. Therefore we have

$$
\begin{gathered}
\lim _{k_{i}^{\prime} \rightarrow \infty} x^{*}\left(\int_{0}^{\infty} \psi(t) L_{k_{i}^{\prime}, t}[f] d t\right)=\lim _{k_{i}^{\prime} \rightarrow \infty} x^{*}\left(\int_{0}^{\omega} \psi(t) L_{k_{i}^{\prime}, t}[f] d t\right) \\
=x^{*}\left(\int_{0}^{\omega} \psi(t) \varphi^{(\omega)}(t) d t\right) .
\end{gathered}
$$

On the other hand, since such $\psi(t)$ belongs to $L_{1}(0, \infty)$,

$$
\lim _{k_{i} \rightarrow \infty} x^{*}\left(T_{k_{i}}(\psi)\right)=\lim _{k_{i} \rightarrow \infty} x^{*}\left(\int_{0}^{\infty} \psi(t) L_{k_{i}}, t[f] d t\right)=x^{*}(T(\psi)) .
$$

Thus

$$
x^{*}\left(\int_{0}^{\omega} \psi(t) \varphi^{(\omega)}(t) d t\right)=x^{*}(T(\psi))
$$

so that

$$
T(\psi)=\int_{0}^{\omega} \psi(t) \varphi^{(\omega)}(t) d t
$$

for every $\psi(t)$ such that $\psi(t) \in L_{2}(0, \omega)$ and $\psi(\mathrm{t}) \equiv 0$ for $t>\omega$. It is easy that if $\omega^{\prime}>\omega$, then $\varphi^{(\omega)}(t)=\varphi^{\left(\omega^{\prime}\right)}(t)$ for almost all $t$ in $(0, \omega)$.

We now define a function $\varphi(t)$ on $(0, \infty)$ into $X$ by

$$
\varphi(t)=\varphi^{(\omega)}(t) \quad \text { for } \omega-1 \leqq t<\varphi \quad(\omega=1,2,3, \ldots) .
$$

From the definition of $\varphi(t)$, it is obvious that $\varphi(t)=\varphi^{(\omega)}(t)$ for almost all $t$ in $(0, \omega), \varphi(t)$ is Bochner measurable and $\|\varphi(t)\|^{2}$ is integrable in any finite interval.

Hence (4. 3) may be written as follows:

$$
T(\psi)=\int_{0}^{\infty} \psi(t) \varphi(t) d t
$$

for every $\psi(t)$ such that $\psi(t) \in L_{2}(0, \omega)$ and $\psi(t)=0$ for $t>\omega$.

Let us put

$$
\psi_{\xi, h}(t)=\left\{\begin{array}{cc}
1 / h & \text { for } \xi \leqq t<\xi+h \\
0 & \text { otherwise }
\end{array}\right.
$$

where $\xi$ and $h$ are any positive number. By (4. 4), we have 


$$
T\left(\psi_{\xi, h}\right)=\frac{1}{h} \int_{\xi}^{\xi+h} \varphi(t) d t
$$

Since $\left\|T\left(\psi_{\xi}, h\right)\right\| \leqq M \int_{0}^{\infty}\left|\psi_{\xi},{ }_{h}(t)\right| d t=M$, we get

$$
\left\|\frac{1}{h} \int_{\xi}^{\xi+h} \varphi(t) d t\right\| \leqq M
$$

Thus

$$
\|\varphi(\xi)\|=\lim _{h \rightarrow 0}\left\|\frac{1}{h} \int_{\xi}^{\xi+h} \varphi(t) d t\right\| \leqq M
$$

for almost all $\xi>0$, so that $\varphi(t)$ is an element in $B_{\infty}([0, \infty) ; X)$.

If we now define the new operator $T^{\prime}$ on $L_{1}(0, \infty)$ into $X$ by

$$
T^{\prime}(\psi)=\int_{0}^{\infty} \psi(t) \varphi(t) d t
$$

where $\psi(t) \in L_{1}(0, \infty)$, then $T^{\prime}$ is a bounded linear operator $L_{1}(0, \infty)$ into $X$. The set $D \equiv \bigcup_{\omega=1}^{\infty}\left\{\psi(t) ; \phi(t) \in L_{2}(0, \omega)\right.$ and $\phi(t)=0$ for $\left.t>\omega\right\}$ is dense in $L_{1}(0, \infty)$ and $T(\psi)=\int_{0}^{\omega=1} \psi(t) \varphi(t) d t=T^{\prime}(\psi)$ for any $\psi \in D$, so that $T=T^{\prime}$.

Thus we get

$$
T(\psi)=\int_{0}^{\infty} \psi(t) \varphi(t) d t
$$

for each $\psi(t) \in L_{1}(0, \infty)$.

Let $\psi(t)=e^{-s t}$. Then, by (4.1) and (4.5), for each $x^{*} \in X^{*}$ and for each $s>0$

$$
\begin{aligned}
x^{*}(f(s)) & =\lim _{k_{i} \rightarrow \infty} x^{*}\left(\int_{0}^{\infty} e^{-s t} L_{k_{i}}, t[f] d t\right) \\
& =\lim _{k_{i} \rightarrow \infty} x^{*}\left(T_{k_{i}}\left(e^{-s t}\right)\right)=x^{*}\left(T\left(e^{-s t}\right)\right) \\
& \left.=x^{*}\left(\int_{0}^{\infty} e^{-s t} \varphi(t) d t\right),\right)
\end{aligned}
$$

so that

$$
f(s)=\int_{0}^{\infty} e^{-s t} \varphi(t) d t \quad(s>0)
$$

Thus the theorem is proved.

Since the above method is quite general, Rooney's result [4; Theorem 9. 2] is also true for a reflexive Banach space. 
Theorem 4 shows that Hille's condition [2; Theorem 10.3.5, (10.3.15)] is also sufficient to represent the function $f(\lambda)$ as a Laplace transform when $X$ is reflexive (= locally weakly compact).

We shall show by an example that the reflexivility is essential for the sufficiency of Theorems 3 and 4 .

Exsample. Let $C[0, \infty]$ be the family of all real-valued continuous functions $x(u)$ of $u$ on the closed interval $[0, \infty]$. The norm of $x(u)$ is defined as the maximum of its absolute value in $[0, \infty] . \quad C[0, \infty]$ is obviously a Banach space. Let $X$ be the family of all bounded linear transformations on $C[0, \infty]$ into itself. It is well known that $X$ is a Banach algebra.

We define

$$
T(t)[x(u)]=e^{-t} x(u+t),
$$$$
(t \geqq 0) \text {. }
$$

Then $\{T(t) ; t \geqq 0\}$ is a semi-group of operators satisfying the following conditions :

$$
\begin{array}{llr}
\left(\mathrm{C}_{1}\right) & T(t) \in X \text { and } T(t+s)=T(t) T(s) & (t, s \geqq 0), \\
\left(\mathrm{C}_{2}\right) & \|T(t)\| \leqq e^{-t} & (t \geqq 0), \\
\left(\mathrm{C}_{3}\right) & \lim _{h \rightarrow 0}\|T(t+h) x-T(t) x\|=0 & (t \geqq 0, x \in C[0, \infty]) .
\end{array}
$$

Furthermore we have

$\left(\mathrm{C}_{4}\right) \quad\|T(t+h)-T(t)\| \geqq e^{-t} \quad(t+h>0, t>0$ and $h \neq 0)$.

In fact, we can always find the element of $C[0, \infty]$ such that $x(t)=1$, $x(t+h)=-1$ and $\max |x(t)|=1$ for any given $t$ and $h$, where $t>0, t+h$ $>0$ and $h \neq 0$. For such an element $x$ we have $\|T(t+h) x-T(t) x\| \geqq e^{-t}$, so that $\left(\mathrm{C}_{4}\right)$ holds.

We denote the infinitesimal generator of $T(t)$ by $A$ and the resolvent of $A$ by $R(s ; A)$. From the theory of semi-group of operators,

$$
R(s ; A) x=\int_{0}^{\infty} e^{-s t} T(t) x d t
$$

for all $s>0$ and for all $x \in C[0, \infty] . R(s ; A)$ has derivatives (strong derivatives in the sense of $X$-norm) of all orders by the resolvent equation $R(s ; A)-R(t ; A)=-(s-t) R(s ; A) R(t ; A) . \quad$ Вy (4.7)

$$
R^{(k)}(s ; A) x=\int_{0}^{\infty}(-t)^{k} e^{-s t} T(t) x d t
$$

and

$$
L_{k, t}[R(\cdot ; A) x]=\frac{1}{k !}\left(\frac{k}{t}\right)^{k+1} \int_{0}^{\infty} u^{k} e^{-k u / t} T(u) x d u,
$$

so that

$$
\frac{s^{k+1}}{k !}\left\|R^{(k)}(s ; A)\right\| \leqq \frac{s^{k+1}}{k !} \int_{0}^{\infty} t^{k} e^{-s t} d t=1 \quad(k=0,1,2, \ldots)
$$


and

$$
\begin{array}{r}
\int_{0}^{\infty}\left\|L_{k}, t[R(\cdot ; A)]\right\|^{p} d t \leqq \int_{0}^{\infty}\|T(u)\|^{p} d u=1 / p \\
\quad(1<p<\infty ; k=1,2, \ldots) .
\end{array}
$$

Thus, the vector-valued function $R(s ; A)$ on $(0, \infty)$ into $X$ satisfies the sufficient conditions of Theorems 3 and 4 .

If there exists an element $\varphi(t)$ of $B_{p}([0, \infty) ; X), 1<p \leqq \infty$, such that

$$
R(s ; A)=\int_{0}^{\infty} e^{-s t} \varphi(t) d t \quad(s>0),
$$

then, by Theorem 1 ,

$$
\lim _{k \rightarrow \infty}\left\|L_{k}, t[R(\cdot ; A)]-\varphi(t)\right\|=0
$$

for almost all $t>0$.

On the other hand, we have from (4. 7), $\left(\mathrm{C}_{3}\right)$ and Theorem 1

$$
\lim _{k \rightarrow \infty}\left\|L_{k, t}[R(\cdot ; A) x]-T(t) x\right\|=0
$$

for all $t>0$ and for all $x \in C[0, \infty]$.

Thus

$$
T(t)=\varphi(t)
$$

for almost all $t>0$, so that $T(t)$ is a Bochner measurable function on the interval $(0, \infty)$ into $X$ such that for $0<t, s<\infty$

$$
T(t+s)=T(t) T(s) \text {. }
$$

Then, by Hille's theorem [2, Theorem 8.3.1],

$$
\lim _{h \rightarrow 0}\|T(t+h)-T(t)\|=0
$$

for all $t>0$. This is contrary to the condition $\left(\mathrm{C}_{4}\right)$. Thus $X$ is not reflexive and $R(s ; A)$ can not be represented as Laplace transformations of function in $B_{p}([0, \infty) ; X)$ for each $p, 1<p \leqq \infty$.

5. Representation of vector-valued functions by Laplace transformations of functions in $B_{1}([0, \infty) ; X)$.

THEOREM 5. Let $X$ be a Banach space. A necessary and sufficient condition that $f(s)$ can be expressed in the form

$$
f(s)=\int_{0}^{\infty} e^{-s t} \varphi(t) d t \quad(s>0),
$$

where $\varphi(t) \in B_{1}([0, \infty) ; X)$, is that

( $\left.i^{\prime \prime}\right) f(s)$ has strong derivatives of all orders in $0<s<\infty$ and $f(\infty)=0$,

$$
\int_{0}^{\infty}\left\|L_{k}, t[f]\right\| d t<\infty \quad(k=1,2, \ldots)
$$


$\left(\mathrm{iii}^{\prime \prime}\right)$

$$
\lim _{j, k \rightarrow \infty} \int_{0}^{\infty}\left\|L_{k}, t[f]-L_{j}, t[f]\right\| d t=0 .
$$

Proof of Sufficiency. By (iii"), there exists an element $\varphi(t)$ of $B_{1}([0$, $\infty) ; X$ ) such that

$$
\lim _{k \rightarrow \infty} \int_{0}^{\infty}\left\|L_{k}, t[f]-\varphi(t)\right\| d t=0 .
$$

Then there exists a positive integer $k_{0}$ such that

$$
\int_{0}^{\infty}\left\|L_{k}, t[f]\right\| d t \leqq 1+\int_{0}^{\infty}\|\varphi(t)\| d t
$$

for $k \geqq k_{0}$.

Let $x^{*}$ be an arbitrary element in $X^{*}$. By Widder's theorem [5; Chap. VII, Theorem 12a], there exists a function of bounded variation $\alpha_{x *}(t)$ such that

$$
x^{*}[f(s)]=\int_{0}^{\infty} e^{-s t} d \alpha_{x *}(t) .
$$

On the other hand, we obtain from the inversion formula

$$
\begin{aligned}
\alpha_{x *}(t)-\alpha_{x *}(0+) & =\lim _{k \rightarrow \infty} \int_{0}^{t} x^{*}\left(L_{k}, u[f]\right) d u \\
& =\int_{0}^{t} x^{*}(\varphi(u)) d u .
\end{aligned}
$$

$f(\infty)=0$ implies $\alpha_{x *}(0+)=0$, so that

$$
\alpha_{x^{*}}(t)=\int_{0}^{t} x^{*}(\varphi(u)) d u .
$$

Thus

$$
x^{*}(f(s))=\int_{0}^{\infty} e^{-s t} x^{*}(\varphi(t)) d t=x^{*}\left(\int_{0}^{\infty} e^{-s t} \varphi(t) d t\right),
$$

so that

$$
f(s)=\int_{0}^{\infty} e^{-s i} \varphi(t) d t \quad(s>0) .
$$

The necessity of the theorem may be proved similarly as in numericallyvalued case. 


\section{REFERENCES}

[1] S. BochNER AND A.E. TAYLOR, Linear functionals on certain spaces of abstractly-valued functions, Ann. of Math., 39(1938), 913-944.

[2] E. Hille, Functional analysis and semi-groups, Amer. Math. Soc. Colloq. Publications, New York(1948).

[3] B. J. PetTis, Differentiation in Banach space, Duke Math. Journ., 5(1939), 254269.

[4] P. G. Rooney, An inversion and representation theory for the Laplace integral of abstractly-valued functions, Canadian Journ. of Math., 5(1954), 190209.

[5] D. V. WidDER, The Laplace transform, Princeton(1941).

Mathematical Institute, ToKyo Metropolitan University.

Added IN Proof. The formula (4.5) can also be obtained from the following theorem.

If $T$ is a bounded linear operator on $L_{1}(0, \infty)$ into a reflexive Banach space $X$, then there exists an element $\varphi(t) \in B_{\infty}([0, \infty) ; X)$ such that

$$
T(\psi)=\int_{0}^{\infty} \psi(t) \varphi(t) d t, \quad \psi(t) \in L_{1}(0, \infty) .
$$

